compared with RAW, $\mathrm{p}<0.05$ Table 2. FITC DEXTRAN uptake (\%) Atorvastatin OxLDL OxLDL+atorvastatin $6 \mathrm{~h} \quad 15.89 \% \pm 0.25 \%$ $18.62 \% \pm 0.45 \% * 18.12 \% \pm 0.76 \% 12 \mathrm{~h} 23.96 \% \pm 1.83 \% \quad 36.50 \% \pm$ $1.27 \% * 29.39 \% \pm 0.50 \% * 24 \mathrm{~h} \quad 25.07 \% \pm 0.76 \% \quad 26.55 \% \pm 0.37$ $30.10 \% \pm 0.21 * \%$ FITC DEXTRAN uptake by RAW is $17.35 \% \pm$ $0.28 \%$; * compared with Atorvastatin, $\mathrm{p}<0.05$ Table 3 . TNF-a (ng/ml) and IL-12 p70 (pg/ml) productin in $24 \mathrm{~h} \mathrm{DMEM+10 \% FCS}$ OxLDL OxLDL+atorvastatin TNF-a $0.142 \pm 0.04 \quad 4.010 \pm 0.34 *$ $1.656 \pm 0.252 *$ IL-12 p70 34.06 $\pm 5.65172 .89 \pm 33.90 * 72.03 \pm 5.62 *$ compared with DMEM+10\% FCS, $\mathrm{p}<0.05$.

Table 1 dendritic cell surface markers expression (\%) in 24 hs

\begin{tabular}{llll}
\hline & RAW & 0xLD & 0xLDL+atorvastatin \\
\hline CD40 & $6.09 \% \pm 0.48 \%$ & $51.68 \% \pm 1.33 \%^{*}$ & $40.09 \% \pm 1.59 \%^{*}$ \\
CD86 & $5.00 \% \pm 0.62 \%$ & $55.04 \% \pm 1.27 \%^{*}$ & $24.29 \% \pm 1.30 \%^{*}$ \\
CD1d & $3.64 \% \pm 0.41 \%$ & $33.79 \% \pm 2.47 \%^{*}$ & $17.10 \% \pm 1.42 \% *$ \\
MHC Class II & $5.09 \% \pm 0.13 \%$ & $33.40 \% \pm 11.54 \%^{*}$ & $22.87 \% \pm 6.665$ \\
\hline
\end{tabular}

${ }^{*}$ compared with RAW, $\mathrm{p}<0.05$.

Table 2 FITC DEXTRAN uptake (\%)

\begin{tabular}{llll}
\hline & Atorvastatin & OxLDL & OxLDL+atorvastatin \\
\hline 6 hs & $15.89 \% \pm 0.25 \%$ & $18.62 \% \pm 0.45 \%^{*}$ & $18.12 \% \pm 0.76 \%$ \\
12 hs & $23.96 \% \pm 1.83 \%$ & $36.50 \% \pm 1.27 \%^{*}$ & $29.39 \% \pm 0.50 \%^{*}$ \\
24 hs & $25.07 \% \pm 0.76 \%$ & $26.55 \% \pm 0.37$ & $30.10 \% \pm 0.21 \%{ }^{*}$ \\
\hline
\end{tabular}

FITC DEXTRAN uptake by RAW is $17.35 \% \pm 0.28 \%$.

${ }^{*}$ compared with Atorvastatin, $\mathrm{p}<0.05$.

Table 3 TNF-a (ng/ml) and IL-12 p70 (pg/ml) productin in $24 \mathrm{hs}$

\begin{tabular}{llll}
\hline & DMEM + 10\%FCS & 0xLDL & 0xLDL+atorvastatin \\
\hline TNF-a & $0.142 \pm 0.04$ & $4.010 \pm 0.34^{*}$ & $1.656 \pm 0.252^{*}$ \\
IL-12 p70 & $34.06 \pm 5.65$ & $172.89 \pm 33.90^{*}$ & $72.03 \pm 5.62$ \\
\hline
\end{tabular}

${ }^{*}$ compared with DMEM+10\%FCS, $p<0.05$.

\section{e0011 THE EFFECT OF SOCS1 SILENCING BY RNA INTERFERENCE ON APOPTOSIS IN HUMAN UMBILICAL VEIN ENDOTHELIAL CELLS}

doi:10.1136/hrt.2010.208967.11

Ding Hao, Li Ju-Xiang, Zhang Ji-Xiang, Dong Jia-Long, Wan Lei, Xia Zi-Rong, Yan Su-Juan, Luo Wei, Cheng Xiao-Shu, Wu Qing-Hua. The Second Affiliated Hospital of Nanchang University (Nanchang China)

Purpose It is well known that apoptosis of endothelial cell (EC) involves the development of atherosclerosis. Down-regulation of SOCS1 (Suppressor of cytokine signalling-1) could induce apoptosis of a variety of cells. However, it has not been reported whether there is any similar phenomenon in EC. Here, we investigated the effect of SOCS1 silencing on Human Umbilical Vein Endothelial Cell (HUVEC) apoptosis induced by hypoxia/reoxygenation and its association with atherosclerosis.

Methods 1. SOCS1 expression in Human Umbilical Vein Endothelial Cell (HUVEC) was determined by RT-PCR and Western Blot. 2. Four different pairs of siRNA (siRNA-1, siRNA-2, siRNA-3, siRNA-4) were designed. 3. The negative control siRNAs with fluorescence were divided into four groups according to different concentrations ( $25 \mathrm{nmol} / \mathrm{l}, 50 \mathrm{nmol} / \mathrm{l}, 75 \mathrm{nmol} / \mathrm{l}, 100 \mathrm{nmol} / \mathrm{l}$ ) and transfected into HUVEC with liposome. Fluorescent microscope was employed to determine the concentration at which siRNAs were transfected most effectively. 4. HUVEC were divided into eight groups: four groups transfected by the different siRNAs already designed, GAPDH positive control group (GAPDH-siRNA-PC), negative control group (siRNA-NC), mock control group (Mock) and blank control group (Blank). These siRNAs were transfected at the optimal concentration. After $48 \mathrm{~h}$, the one that most extremely silenced SOCS1 was selected by RT-PCR and Western Blot. 5. The selected siRNA as well as siRNA-NC was transfected at the optimal concentration. After $24 \mathrm{~h}, \mathrm{HUVEC}$ were divided into four groups: a. siRNA-NC; b. siRNA-NC+hypoxia/reoxygenation; c. SOCS1 siRNA; d. SOCS1 siRNA+hypoxia/reoxygenation. Then, the expressions of Caspase -3 and Bax were detected by Western Blot, and the apoptosis rates were assessed by flow cytometry.

Results 1 . It was $50 \mathrm{nmol} / \mathrm{l}$ at which siRNAs were transfected most effectively. 2. The expressions of SOCS1 in siRNA-1, siRNA-2, siRNA-3 and siRNA-4 groups declined compared to four control groups $(p<0.05)$. siRNA -3 had the optimal silencing efficiency in four different pairs of siRNA $(p<0.05)$. 3. After RNAi and hypoxia/ reoxygenation, the expressions of Caspase -3 and Bax in SOCS1 siRNA+hypoxia/reoxygenation group elevated compared to other three groups $(p<0.05)$. The expressions of Caspase -3 and Bax in siRNA-NC+hypoxia/reoxygenation group increased compared to siRNA-NC group $(p<0.05)$. The expression of Caspase-3 in siRNA$\mathrm{NC}+$ hypoxia/reoxygenation group increased compared to SOCS1 siRNA group $(p<0.05)$. There was no statistical significance of Caspase -3 between siRNA-NC and SOCS1 siRNA groups ( $p>0.05)$. Bax in SOCS1 siRNA group was higher than that in siRNA-NC group $(p<0.05)$. 4. After RNAi and hypoxia/reoxygenation, the apoptosis rate of SOCS1 siRNA+hypoxia/reoxygenation group increased compared to other three groups $(p<0.05)$. The apoptosis rate of siRNA-NC+hypoxia/reoxygenation group increased compared to siRNA-NC and SOCS1 siRNA groups $(p<0.05)$. There was no statistical significance between siRNA-NC and SOCS1 siRNA groups ( $p>0.05)$.

Conclusion 1. The expression of SOCS1 in EC could be inhibited effectively by RNAi. 2. SOCS1 silencing could exacerbate EC apoptosis induced by hypoxia/reoxygenation.

\section{E0012 APELIN STIMULATES GLUCOSE UPTAKE THROUGH PI3KAKT PATHWAY IN INSULIN RESISTANT 3T3L1 ADIPOCYTES}

doi:10.1136/hrt.2010.208967.12

Zhu Shunming, Cao Feng, Li Weijie, Wang Chen, Wang Yabin, Liang Dong, Wang Haichang. Xijing Hospital

Background Apelin, a cytokine mainly secreted by adipocytes, shows beneficial effect on insulin resistance. However, the molecular mechanism underlying is still poorly understood. This study was to investigate the mechanisms of apelin on insulin resistant improvement in 3T3-L1 adipocytes.

Methods Insulin resistance in 3T3-L1 adipocytes was induced by TNF- $\alpha$. The effects of apelin on glucose metabolism were investigated by 3H-2-Deoxy-glucose uptake. The effects of apelin on glucose transporter four translocation were visualised by immunofluorescence microscopy. The role of PI3K/Akt pathway was assessed by immunoblotting. The effects of apelin on interlukin-6 and adiponectin mRNA expression were observed by RT-PCR.

Results Insulin-stimulated glucose uptake could be reduced by $47 \%$ by treatment with TNF- $\alpha$ for $24 \mathrm{~h}$. Apelin could improve insulinstimulated glucose uptake with the involvement of PI3K/Akt pathway. In addition, microscopy imaging showed apelin could increase GLUT4 translocation to plasma membrane. RT-PCR assay indicated apelin also increased adiponectin while reduced interlukin-6 mRNA expression in insulin-resistant adipocytes.

Conclusion This study suggested that apelin stimulates glucose uptake through PI3K/Akt pathway and GLUT4 translocation, while moderate inflammatory responses in insulin-resistant 3T3-L1 adipocytes. 